Rev. salud pública. Sup. 8 (1): 86-101, 2006

\title{
Resistencia Antimicrobiana en Unidades de Cuidado Intensivo de Bogotá, Colombia, 2001-2003
}

\author{
Carlos Alvarez ${ }^{1}$, Jorge Cortes ${ }^{2}$, Álvaro Arango ${ }^{3}$, Constanza Correa ${ }^{4}$, Aura Leal ${ }^{5}$ y \\ Grebo ${ }^{1}$ \\ ${ }^{1}$ Médico. Especialista en Infectología. Unidad de Infectología, Hospital Universitario San Ignacio. \\ Facultad de Medicina, Universidad Nacional de Colombia. E-mail: caalvarezmo@unal.edu.co \\ 2 Médico. Especialista en Infectología. Hospital Universitario San Ignacio. Instituto Nacional de \\ Cancerología. E-mail: jorgecortes@yahoo.com \\ ${ }^{3}$ Médico. Especialista en Infectología. Fundación Cardioinfantil. E-mail: aarangod@sky.net.co \\ ${ }^{4}$ Bacterióloga. Hospital Simón Bolívar. \\ E-mail: rmendez@hotmail.com \\ ${ }^{5}$ Médico. M. Sc. Control de Enfermedades Infecciosas. Especialista en Microbiología y Parasitología \\ Médicas. Departamento de Microbiología, Facultad de Medicina. Universidad Nacional de Colombia. \\ E-mail: allealc@unal.edu.co
}

Recibido 28 Septiembre 2005/Enviado para Modificación 5 Marzo 2006/Aceptado 15 Abril 2006

\section{RESUMEN}

Objetivos Determinar la resistencia antimicrobiana entre los aislamientos bacterianos identificados en pacientes hospitalizados en Unidades de Cuidado Intensivo.

Métodos Se recolectó la información de los aislamientos de microbiología provenientes de las Unidades de Cuidado Intensivo (UCl) de 14 instituciones de tercer nivel, pertenecientes al Grupo para el Control de la Resistencia Bacteriana de Bogotá (GREBO), entre los años 2001 y 2003. Se obtuvieron 27301 aislamientos y su perfil de susceptibilidad fue analizado por el programa Whonet 5.3.

Resultados Los microorganismos aislados con mayor frecuencia fueron Staphylococcus aureus, Escherichia coli, Staphylococci coagulasa negativo (SCN), Pseudomonas aeruginosa y Klebsiella pneumoniae. Las tasas de resistencia de S. aureus y de SCN a oxacilina del 2001 al 2003 oscilaron entre 61 y $63 \%$ y entre 78 y $83 \%$, respectivamente. La resistencia a cefalosporinas de tercera generación en E. coli fue cercana a $10 \%$ y a ciprofloxacina fue superior a $20 \%$. La resistencia a cefalosporinas de tercera generación de K. pneumoniae fue superior a $30 \%$ durante el 2001. La resistencia

\footnotetext{
${ }^{1}$ Grupo para el Control de la Resistencia Bacteriana de Bogotá
} 
de $P$. aeruginosa a varios grupos de antibióticos superó $30 \%$ y la frecuencia de cepas multirresistentes osciló entre 16 y $24 \%$.

Conclusión Las tasas de resistencia encontradas son superiores a las mostradas por estudios de vigilancia en E.E.U.U., Europa y otras ciudades de América Latina.

Palabras Clave: Colombia, epidemiología, resistencia microbiana a las drogas, unidad de cuidado intensivo, vigilancia de la población (fuente: DeCS, BIREME).

\section{ABSTRACT}

Anti-microbial resistance in Intensive Care Units in Bogotá, Colombia, 2001-2003

Objectives Determining the frequency of antimicrobial resistance amongst bacterial isolates obtained from patients in Intensive Care Units (ICU).

Methods Study data relating to 2001 to 2003 regarding microbiological isolates was obtained from a laboratory network for the ICUs of 14 third-level hospitals in Bogotá belonging to the Bogotá Bacterial Resistance Control Group (BBRCG). 27,301 isolates were obtained and their susceptibility profiles were analysed using WHONET 5.3.

Results Staphylococcus aureus, Escherichia coli, coagulase negative Staphylococci (CNS), Pseudomonas aeruginosa y Klebsiella pneumoniae were the most commonly found microorganisms. Oxacillin-resistance rate ranged from $61 \%$ at $63 \%$ during 2001 to 2003 amongst S. aureus isolates and $78 \%$ to $83 \%$ amongst coagulase negative Staphylococci (CNS). E. coli resistance to third generation cephalosporines was found to be about $10 \%$ and over $20 \%$ to ciprofloxacin. K. pneumoniae isolates' resistance to thirdgeneration cephalosporines was found to be more than $30 \%$ in 2001. $P$. aeruginosa resistance to all but a few antibiotics was found to be over $30 \%$ and multirresistance frequency was found to be $16 \%$ to $24 \%$.

Conclusion The antimicrobial resistance rates found in the ICU of hospitals in Bogotá were higher than those reported in surveillance studies in the USA, Europe or other Latin-American cities.

Key Words: Colombia, epidemiology, Drug Resistance, Microbial, Intensive Care Units, population surveillance (source: $M e S H, N L M$ ).

L a resistencia antimicrobiana (RA) ha sido considerada un fenómeno emergente en todo el mundo, especialmente en los hospitales. Los datos publicados en estudios multicéntricos de Estados Unidos, Europa y América Latina muestran un nivel de resistencia creciente en bacterias aisladas en los hospitales (1). En Latinoamérica y, especialmente en Colombia, se dispone de un número limitado de datos acerca de la magnitud 
de este problema, debido al escaso número de hospitales que colaboraron en estudios multinacionales o a su publicación en boletines de distribución local $(2,3)$.

En los hospitales, las Unidades de Cuidado Intensivo (UCI), debido a sus características permiten la concentración de los factores que conllevan a la RA (4): uso frecuente de antibióticos de amplio espectro (5), uso de procedimientos y dispositivos invasivos, , pacientes con alta frecuencia de comorbilidad y estancias prolongadas (6,7), entre otras. Por tanto, es frecuente observar mayores tasas de RA en los aislamientos de microorganismos provenientes de estas unidades.

El propósito de este artículo es describir los resultados del programa de vigilancia GREBO, a partir de los aislamientos realizados en las UCI de 14 hospitales de tercer nivel de Bogotá, Colombia, entre el año 2001 y el 2003.

\section{MÉTODOS}

Hospitales participantes

Se recolectó la información procedente de pacientes atendidos en las UCI de 14 hospitales. Las instituciones participantes fueron: Clínica del Niño (6 camas de UCI pediátrica), Fundación San Carlos (42 camas de UCI adultos médico quirúrgica, Cardiovascular y pediátrica), Hospital El Tunal (20 camas de UCI médico-quirúrgica), Hospital del Occidente Kennedy (43 camas de UCI médico-quirúrgica, pediátrica), Clínica de Occidente (40 camas de UCI adultos, médico quirúrgica y cardiovascular), Hospital Militar Central (28 camas de UCI médica, quirúrgica y cardiovascular), Hospital Simón Bolívar (54 camas de UCI médico quirúrgica, pediátrica, neonatal y quemados), Hospital Universitario San Ignacio (52 camas de UCI adultos, médico quirúrgica, cardiovascular, pediátrica y neonatal), Hospital San José (22 camas de UCI adultos, médico quirúrgica, cardiovascular y pediátrica), Hospital Universitario Clínica San Rafael (41 camas de UCI adultos, médico quirúrgica, cardiovascular, pediátrica y neonatal), Instituto Nacional de Cancerológica (6 camas de UCI adultos y médico quirúrgica), Clínica San Pedro Claver (28 camas de UCI médico quirúrgica y cardiovascular), Hospital Santa Clara (24 camas de UCI médico quirúrgica), Fundación Cardio Infantil (54 camas de UCI adultos, médico quirúrgica, cardiovascular y pediátrica).

Recolección de datos 
Cada 3 a 6 meses los laboratorios de microbiología enviaron los informes de susceptibilidad antimicrobiana, los cuales fueron incorporados a una base de datos creada en Whonet (Organización Mundial de la Salud - OMS, versión 5.3) utilizando el programa BackLink 2 (OMS) para transferir la información de los sistemas automatizados VITEK ${ }^{\circledR}$ (Biomerieux, Paris, Fr) y MicroScan ${ }^{\circledR}$ (Dade Behring, Sacramento, EEUU). En los laboratorios con sistemas manuales (Kirby Bauer), la información recolectada fue incluida directamente al programa Whonet. En este caso se incluyeron sólo la información referente a los aislamientos provenientes de UCI y se eliminaron los aislamientos repetidos, es decir aquellos gérmenes aislados del mismo sitio de un paciente en la misma fecha.

Susceptibilidad antimicrobiana y control de calidad

La información de los resultados de susceptibilidad antimicrobiana fue clasificada como sensible, intermedia o resistente de acuerdo a los puntos de corte utilizados para cada antibiótico determinado a partir de las recomendaciones de la NCCLS (2003). Para el análisis se consideró Pseudomonas multirresistente, a aquellos aislamientos de Pseudomonas spp. resistentes a cuatro o más antibióticos (piperacilina o piperacilina/tazobactam, ceftazidima, imipenem o meropenem, ciprofloxacina y amikacina) (8), posible fenotipo Amp C, a los aislamientos de enterobacterias corresistentes a cefamicinas (cefotetan) y oximinocefalosporinas (ceftriaxona ó Ceftazidima); posible fenotipo de betalactamasa de espectro extendido (BLEE) a los aislamientos de Escherichia coli, Klebsiella spp. y Enterobacter spp. con sensibilidad a una cefamicina (cefotetan) y resistencia a una cefalosporina de tercera generación o aztreonam. Los laboratorios incluidos en el programa Whonet, fueron sometidos a un programa de calidad externo, llevado a cabo por el laboratorio de Microbiología del Instituto Nacional de Salud como previamente se describió (9).

\section{Análisis estadístico}

Los resultados de susceptibilidad antimicrobiana fueron comparados por distribuciones de frecuencia. Usando el programa EPIINFO 6.04 se compararon los porcentajes, medias, medianas y percentiles, usando las pruebas de chi cuadrado o prueba exacta de Fisher, cuando fue necesario, considerando valores significativos con $\mathrm{p}<0.05$. Para realizar el análisis de corresistencia entre varios antibióticos se utilizó la función "scatterplot" del programa Whonet (OMS versión 5.3). 


\section{RESULTADOS}

Frecuencia de los aislamientos microbianos y sitio de origen

Se identificaron 18407 microorganismos durante los 3 años en las UCI de los 14 hospitales participantes, predominando los gérmenes Gram negativos $(49,2 \%)$ mientras que los hongos ocuparon un porcentaje bajo (7,1 \%). En la Tabla 1 se describen los microorganismos aislados con mayor frecuencia de acuerdo al sitio de aislamiento. En general Staphylococcus aureus y Escherichia coli fueron los gérmenes aislados con mayor frecuencia. Otros microorganismos de importancia epidemiológica en UCI, como Serratia marcescens, Enterococcus faecium, Stenotrophomonas maltophilia, Streptococcus pneumoniae fueron aislados con muy baja frecuencia: 348, 1,9 \%; 185, 1 $\%$; 163, 0,9 \%; 189, $1 \%$, respectivamente. A su vez, los sitios en los que se encontraron mayor número de aislamientos, fueron sangre, secreciones de heridas, orina y catéteres ( $23 \%, 17,8 \%, 11,2 \%, 10,8 \%)$, mientras que aislamientos de líquido cefalorraquídeo sólo ocuparon un 0,6 \% del total.

Tabla 1. Microorganismos más frecuentes según el sitio de aislamiento en UCI de

\begin{tabular}{|c|c|c|c|c|c|c|c|c|c|c|}
\hline \multicolumn{11}{|c|}{ Bogotá, 2001 a 2003} \\
\hline \multirow{3}{*}{ Microorganismo } & \multicolumn{2}{|c|}{ TOTAL } & \multicolumn{2}{|c|}{ A } & \multicolumn{2}{|c|}{ B } & \multicolumn{2}{|c|}{ C } & \multicolumn{2}{|c|}{$\mathrm{D}$} \\
\hline & No & $\%$ & No & $\%$ & No & $\%$ & No & $\%$ & No & $\%$ \\
\hline & 18407 & 100 & 3078 & 100 & 1497 & 100 & 1104 & 100 & 736 & 100 \\
\hline S. aureus & 2560 & 13,8 & 481 & 12 & 34 & 2 & 208 & 11,1 & 277 & 27,2 \\
\hline E. coli & 1926 & 10,4 & 164 & 4,1 & 486 & 28,1 & 414 & 22 & 58 & 5,7 \\
\hline S. epidermidis & 1749 & 9,4 & 876 & 21,9 & 17 & 1 & 89 & 4,7 & 22 & 2,2 \\
\hline K. pneumoniae & 1267 & 6,8 & 209 & 5,2 & 135 & 7,8 & 99 & 5,3 & 98 & 9,6 \\
\hline$P$. aeruginosa & 1254 & 6,8 & 116 & 2,9 & 119 & 6,9 & 179 & 9,5 & 110 & 10,8 \\
\hline A. baumannii & 852 & 4,6 & 152 & 3,8 & 25 & 1,5 & 87 & 4,6 & 77 & 7,6 \\
\hline E. faecalis & 787 & 4,3 & 143 & 3,6 & 85 & 4,9 & 138 & 7,4 & 17 & 1,7 \\
\hline C. albicans & 719 & 3,9 & 84 & 2,1 & 210 & 12,2 & 50 & 2,66 & 34 & 3,3 \\
\hline E. cloacae & 714 & 3,8 & 99 & 2,5 & 67 & 3,9 & 66 & 3,51 & 36 & 3,5 \\
\hline SCN & 662 & 3,6 & 184 & 4,6 & 3 & 0,17 & 28 & 1,49 & 7 & 0,7 \\
\hline
\end{tabular}

A: Sangre; B: Orina; C: Liquido Abdominal; D: Secreción traqueal; SCN: Staphylococcus, coagulase negativa

Resistencia entre cocos Gram positivos

La Tabla 2 muestra la alta resistencia tanto del S. aureus como de los SCN, resaltando la ausencia cepas con sensibilidad disminuida o resistencia a vancomicina. En $S$. aureus se encontró un aumento en la resistencia a oxacilina entre el año 2001 y 2002 con una disminución para el 2003 (estas diferencias no fueron estadísticamente significativa con un valor de $\mathrm{p}>0.05$ ). La frecuencia de corresistencia en aislamientos de $S$. aureus resistente a la oxaci- 
lina fue de 59,8 \% para clindamicina, 57,4 \% para eritromicina, 59,5 \% para quinolonas, 8,0 \% para tetraciclina, 3,9 \% para rifampicina y 4,5\% para trimetoprim/sulfametoxazol.En SCN se observó un aumento de la sensibilidad frente a oxacilina al comparar el 2001 con el 2002 y $2003(\mathrm{p}<0.05)$. Para los otros antibióticos también se observó un amento progresivo en la susceptibilidad en el periodo estudiado.

La sensibilidad de E. faecalis frente a ampicilina y vancomicina se ha mantenido cercana a 95 \% durante los años 2001 y 2002 (Tabla 3), con un aumento estadísticamente significativo de la sensibilidad en el año 2003 $(\mathrm{p}<0.5)$. Por el contrario, la resistencia de E. faecium frente a ampicilina y vancomicina, aumentó en el 2002 y disminuyó nuevamente en el 2003. Los aislamientos resistentes a vancomicina (EVR), aislados entre 2001 y 2002, se encontraron en solo 5 hospitales participantes.

Tabla 2. Resistencia antimicrobiana de especies de Staphylococci

\begin{tabular}{|c|c|c|c|c|c|c|c|c|c|c|c|c|}
\hline \multirow{3}{*}{ Antibiótico } & \multicolumn{6}{|c|}{ S. aureus } & \multicolumn{6}{|c|}{$\mathrm{SCN}$} \\
\hline & \multicolumn{2}{|c|}{2001} & \multicolumn{2}{|c|}{2002} & \multicolumn{2}{|c|}{2003} & \multicolumn{2}{|c|}{2001} & \multicolumn{2}{|c|}{2002} & \multicolumn{2}{|c|}{2003} \\
\hline & $n$ & $\%$ & $n$ & $\%$ & $\mathrm{n}$ & $\%$ & $\mathrm{n}$ & $\%$ & $n$ & $\%$ & $n$ & $\%$ \\
\hline Ciprofloxacina & 526 & 58,4 & 778 & 62,9 & 802 & 59,7 & 800 & 57,5 & 807 & 51,9 & 1099 & 47,1 \\
\hline Clindamicina & 569 & 62,2 & 895 & 62,6 & 944 & 59,2 & 843 & 74,6 & 981 & 69,2 & 1205 & 63 \\
\hline Eritromicina & 505 & 62,6 & 768 & 60,7 & 850 & 60,2 & 785 & 70,1 & 889 & 70 & 1164 & 68,7 \\
\hline Gentamicina & 491 & 58,7 & 764 & 61,5 & 814 & 59,7 & 781 & 62,2 & 891 & 60,9 & 1125 & 53,2 \\
\hline Oxacilina & 583 & 61,2 & 909 & 62,9 & 946 & 60,6 & 865 & 83,1 & 994 & 79,4 & 1210 & 78,3 \\
\hline Rifampicina & 487 & 9,9 & 730 & 2,6 & 821 & 3,7 & 764 & 25,4 & 774 & 19,4 & 1093 & 17,1 \\
\hline Tetraciclina & 487 & 25,1 & 738 & 11,8 & 788 & 12,3 & 764 & 29,6 & 871 & 28,5 & 1111 & 30,3 \\
\hline Trimetoprim/Sulfametoxazol & 534 & 11 & 868 & 3,6 & 924 & 4,8 & 811 & 50,1 & 928 & 45,3 & 1198 & 47,4 \\
\hline Vancomicina & 578 & 0 & 906 & 0 & 936 & 0 & 865 & 0 & 992 & 0 & 1209 & 0 \\
\hline
\end{tabular}

Tabla 3. Resistencia antimicrobiana de especies de Enterococcus

\begin{tabular}{|c|c|c|c|c|c|c|c|c|c|c|c|c|}
\hline \multirow{3}{*}{ Antibiótico } & \multicolumn{6}{|c|}{ E. faecalis } & \multicolumn{6}{|c|}{ E. faecium } \\
\hline & \multicolumn{2}{|c|}{2001} & \multicolumn{2}{|c|}{2002} & \multicolumn{2}{|c|}{2003} & \multicolumn{2}{|c|}{2001} & \multicolumn{2}{|c|}{2002} & \multicolumn{2}{|c|}{2003} \\
\hline & $\mathrm{n}$ & $\%$ & $\mathrm{n}$ & $\%$ & $\mathrm{n}$ & $\%$ & $\mathrm{n}$ & $\%$ & $\mathrm{n}$ & $\%$ & $\mathrm{n}$ & $\%$ \\
\hline Ampicilina & 246 & 5,7 & 253 & 4,7 & 253 & 2,4 & 50 & 50 & 66 & 57,6 & 58 & 44,8 \\
\hline Ciprofloxacina & 221 & 37,1 & 238 & 33,6 & 253 & 24,9 & 52 & 44,2 & 64 & 43,8 & 59 & 37,3 \\
\hline $\begin{array}{l}\text { Gentamicina-Alta } \\
\text { Carga }\end{array}$ & 187 & 28,9 & 186 & 27,4 & 243 & 21,4 & 43 & 16,3 & 53 & 28,3 & 52 & 30,8 \\
\hline Imipenem & 195 & 6,2 & 185 & 4,9 & 182 & 2,2 & 45 & 15,6 & 27 & 18,5 & 16 & 0 \\
\hline Nitrofurantoina & 218 & 3,7 & 219 & 1,8 & 237 & 1,7 & 52 & 7,7 & 63 & 6,3 & 51 & 5,9 \\
\hline Vancomicina & 251 & 2 & 266 & 4,1 & 269 & 3 & 53 & 13,2 & 67 & 17,9 & 59 & 0 \\
\hline
\end{tabular}


Resistencia de bacilos entéricos

En la Tabla 4 se observa la susceptibilidad antimicrobiana en los aislamientos de E. coli, K. pneumoniae y Enterobacter cloacae. Al hacer un análisis de la corresistencia se encontró que en 257 aislamientos de E. coli probados contra ceftriaxona y cefotetan, solo $0,8 \%$ mostraron corresistencia a las dos moléculas, mientras que $8 \%$ mostraron un posible fenotipo BLEE. Al evaluar 200 aislamientos de K. pneumoniae, se encontró corresistencia en 3,5 \% entre los dos antibióticos mientras que 19,5 \% mostraron un posible fenotipo BLEE. En E. cloacae los valores fueron de 39,5 \% para un posible fenotipo AmpC) y 4,8 \% para un posible fenotipo BLEE. La susceptibilidad a los carbapenémicos se mantuvo por encima de $95 \%$ para todos los aislamientos durante los tres años mientras que a las quinolonas ha aumentado en relación al 2001, especialmente entre los aislamientos de K. pneumoniae $(\mathrm{p}<0.01)$. La resistencia a la gentamicina fue inferior $20 \%$ entre los aislamientos de $E$. coli durante los 3 años, disminuyó en $K$. pneumoniae y se mantuvo en $E$. cloacae ( $>0.05$ para las diferencias entre los 3 años). La sensibilidad frente a amikacina fue mayor para los 3 años en $E$. coli y menor en $K$. pneumoniae y E. cloacae.

Tabla 4. Resistencia antimicrobiana en E. coli, K. pneumoniae y E. cloacae

\begin{tabular}{|c|c|c|c|c|c|c|}
\hline \multirow{3}{*}{ Antibiótico } & \multicolumn{6}{|c|}{$2001-2003$} \\
\hline & \multicolumn{2}{|c|}{ E. coli } & \multicolumn{2}{|c|}{ K. pneumoniae } & \multicolumn{2}{|c|}{ E. cloacae } \\
\hline & $\mathrm{n}$ & $\%$ & $n$ & $\%$ & $\mathrm{n}$ & $\%$ \\
\hline Amikacina & 1651 & 6,2 & 1130 & 16,3 & 644 & 33,4 \\
\hline Ampicilina/Sulbactam & 1539 & 44,9 & 1023 & 47,9 & 615 & 72,2 \\
\hline Aztreonam & 961 & 12,1 & 662 & 40 & 333 & 51,7 \\
\hline Cefazolina & 1420 & 24,6 & 955 & 48,3 & 560 & 95,5 \\
\hline Cefepime & 839 & 4,4 & 564 & 15,4 & 324 & 30,6 \\
\hline Cefotaxima & 829 & 6,9 & 637 & 22,8 & 409 & 51,8 \\
\hline Ceftriaxona & 1490 & 7,8 & 1023 & 34,7 & 581 & 52,7 \\
\hline Ciprofloxacina & 1745 & 22,2 & 1155 & 11,4 & 661 & 36,3 \\
\hline Imipenem & 1405 & 1,2 & 1031 & 1,6 & 613 & 4,1 \\
\hline Levofloxacina & 751 & 20,8 & 445 & 7,4 & 230 & 39,6 \\
\hline Meropenem & 470 & 1,1 & 307 & 2 & 167 & 3,6 \\
\hline Piperacilina/Tazobactam & 750 & 6,1 & 532 & 24,1 & 320 & 36,9 \\
\hline Trimetoprim/Sulfametoxazol & 1648 & 46,2 & 1097 & 36,9 & 646 & 37,5 \\
\hline
\end{tabular}


La resistencia de $P$. aeruginosa a los betalactámicos, quinolonas y aminoglucósidos se mantuvo elevada durante los 3 años mientras que para cefepime y ciprofloxacina disminuyó su sensibilidad en el año 2002 pero aumentó en el 2003. En los carbapenémicos se observó un aumento progresivo de la sensibilidad del imipenem durante los 3 años, encontrándose una diferencia significativa $(\mathrm{p}<0.05)$ entre los años 2001 y 2003, mientras que en meropenem disminuyó para los 2 años de los que se dispone de aislamientos probados $(\mathrm{p}<0.05)$. El porcentaje de aislamientos multiresistentes fue de 23 $\%$ en el 2001, $24 \%$ en el 2002 y de $16 \%$ en el 2003 ( $<<0.05$ para la diferencia entre el 2002 y el 2003). En aislamientos de A. baumanni la resistencia al imipenem se incrementó de forma importante entre estos años $(p<0.001)$, mientras que para ampicilina/sulbactam, a pesar de ser elevada se mantuvo estable. La frecuencia de aislamientos coresistentes a una cefalosporina de tercera generación y al imipenem creció de forma significativa durante los 3 años observados (1,4 \%, 12 \% y 15,7 \% para 2001, 2002 y 2003 respectivamente, $\mathrm{p}<0.05$ para la diferencia entre los 3 años, excepto entre 2002 y 2003).

Tabla 5. Resistencia antimicrobiana en Pseudomonas aeruginosa y Acinetobacter baumanni

\begin{tabular}{|c|c|c|c|c|c|c|c|c|c|c|c|c|}
\hline \multirow{3}{*}{ Antibiótico } & \multicolumn{6}{|c|}{ P. aeruginosa } & \multicolumn{6}{|c|}{ A. baumanni } \\
\hline & \multicolumn{2}{|c|}{2001} & \multicolumn{2}{|c|}{2002} & \multicolumn{2}{|c|}{2003} & \multicolumn{2}{|c|}{2001} & \multicolumn{2}{|c|}{2002} & \multicolumn{2}{|c|}{2003} \\
\hline & $\mathrm{n}$ & $\%$ & $\mathrm{n}$ & $\%$ & $\mathrm{n}$ & $\%$ & $\mathrm{n}$ & $\%$ & $\mathrm{n}$ & $\%$ & $\mathrm{n}$ & $\%$ \\
\hline Amikacina & 388 & 26,8 & 398 & 30,4 & 355 & 22,3 & 247 & 34,4 & 262 & 60,3 & 276 & 65,2 \\
\hline $\begin{array}{l}\text { Ampicilina/ } \\
\text { Sulbactam }\end{array}$ & & & & & & & 223 & 37,7 & 245 & 35,1 & 254 & 34,3 \\
\hline Aztreonam & 329 & 44,7 & 126 & 29,4 & 203 & 17,2 & 212 & 76,9 & 135 & 56,3 & 159 & 54,7 \\
\hline Cefepime & 46 & 13 & 218 & 30,7 & 308 & 14,3 & 36 & 58,3 & 158 & 65,8 & 249 & 69,9 \\
\hline Ceftazidima & 413 & 40,2 & 391 & 35,3 & 321 & 17,8 & 255 & 36,9 & 275 & 27,6 & 263 & 27,4 \\
\hline Ciprofloxacina & 392 & 55,4 & 394 & 58,4 & 361 & 40,4 & 241 & 75,1 & 277 & 77,6 & 267 & 75,3 \\
\hline Gentamicina & 369 & 56,9 & 375 & 54,9 & 340 & 41,5 & 228 & 78,5 & 262 & 83,2 & 265 & 82,6 \\
\hline Imipenem & 380 & 31,1 & 351 & 30,5 & 354 & 26 & 234 & 11,5 & 275 & 37,8 & 260 & 48,1 \\
\hline Meropenem & & & 41 & 12,2 & 259 & 23,9 & & & 19 & 31,6 & 198 & 52,0 \\
\hline $\begin{array}{l}\text { Piperacilina/ } \\
\text { Tazobactam }\end{array}$ & 43 & 11,6 & 192 & 29,7 & 282 & 12,1 & 33 & 69,7 & 86 & 45,3 & 141 & 22,7 \\
\hline
\end{tabular}

\section{DISCUSIÓN}

La vigilancia de la resistencia antimicrobiana se ha convertido en uno de los pasos fundamentales para combatir este fenómeno emergente, que ya se con- 
sidera un problema de salud pública a nivel mundial (10). En la UCI está información es más valiosa por cuanto los patrones de resistencia difieren de los encontrados en los mismos hospitales y en la comunidad $(11,12)$. La frecuencia de gérmenes encontrada por nosotros es muy similar a la descrita por Fluit, en UCI de Europa, y a la de Streit., en EEUU (13), con la excepción de la menor prevalencia de $P$. aeruginosa (6,75 \% vs $12,5 \%$ y $12,2 \%$, respectivamente) con un aumento concomitante en los SCN (13,2 \% vs 10,5 $\%$ y 7,0 \%). Estas diferencias probablemente son explicadas porque incluimos gérmenes no solo causantes de infección sino también de colonizaciones mientras que Fluit y Streit, solo analizaron aquellos asociados a infección. Sin embargo, dado que las principales infecciones nosocomiales observadas en la UCI son las asociadas a catéteres, pulmón, tracto urinario y las del sitio operatorio $(14,15)$, en nuestro estudio los aislamientos procedentes de dichos sitios anatómicos superan el $60 \%$ y nos permite explicar las frecuencias encontradas de S. aureus, SCN, E. coli, Klebsiella spp. y P. aeruginosa en las UCI de la ciudad, gérmenes tradicionalmente causantes de este tipo de infecciones.

El principal hallazgo de este estudio es la confirmación de las elevadas tasas de RA entre los distintos grupos de microorganismos. En el estudio de Arias se encontró en el año 2001 una resistencia de $S$. aureus a la oxacilina del $52 \%$ y una resistencia de SCN a la oxacilina de 73\% (16). En este estudio las tasas fueron mayores y en general la resistencia a la oxacilina fue más alta en SCN al compararse con S. aureus con una corresistencia a los otros antibióticos con actividad antiestafilocóccica elevada, lo que podría explicarse en parte, por la mayor presión generada por el mayor uso de antibióticos y la transmisión cruzada. Las diferencias con nuestros resultados pueden ser explicadas por su origen, ya que generalmente los perfiles de resistencia en UCI son mayores como se ha descrito previamente y confirmado por GREBO (9). Sin embargo, al comparar estas tasas de resistencia con las informadas en UCI de Asia, Europa y los Estados Unidos, nuestras tasas son superiores o similares, con excepción de la resistencia de los Enterococci a la vancomicina, la cual fue inferior (12,13,17-19). Con respecto a América Latina los perfiles de resistencia de E. coli, K. pneumoniae y E. cloacae son similares a los encontrados por Mendes en Brasil, pero los de P. aeruginosa para carbapenémicos muy superiores (40,8 - 36,8 vs. 18,6 \%) (20). Estas diferencias con nuestros hospitales reflejan la importancia de disponer de datos locales que permitan tomar mejores decisiones clínicas o establecer políticas de tratamiento, de control de infecciones y de uso prudente de antibióticos.

Es de resaltar el hecho de que la resistencia a la vancomicina entre los aislamientos de E. faecium haya disminuido considerablemente en las UCI 
de Bogotá durante el último año, aunque no es claro cual es el factor determinante de este fenómeno. El estudio multicéntrico previamente mencionado (16) mostró una alta tasa de resistencia entre los años 2001 y 2002, que nuevamente puede ser explicado por el tipo de muestreo empleado, pero también se evidenció que las cepas identificadas a las que se les realizó estudio molecular estaban altamente relacionadas. Por lo que podemos estar en frente a brotes que se desarrollaron en algunos de los hospitales participantes, hecho que concuerda con que solo 5 hospitales reportaran EVR y 2 de ellos informaran 87 \% de las muestras resistentes. Ello explicaría las altas tasas observadas, junto con la posterior desaparición de este tipo de aislamientos en la UCI. En Medellín se observó un brote de características clonales con la expresión de Van A que ha persistido en el tiempo (21).

Los niveles de resistencia a las cefalosporinas entre las enterobacterias se han incrementado en los últimos años. Un estudio realizado en varios centros hospitalarios del país en 1997 mostró una resistencia a las cefalosporinas de tercera generación en E. coli menor a 7 \% (22), en Klebsiella spp. entre 13 y $26 \%$ y en $E$. cloacae entre 18 y $29 \%$. En nuestro estudio la resistencia a las cefalosporinas de tercera generación y aztreonam se ha mantenido en niveles entre 7,1 y $13,5 \%$ en $E$. coli, 20,1 y $52,8 \%$ en $K$. pneumoniae y 40,9 y 57,8 $\%$ en E. cloacae. Estos niveles de resistencia pueden deberse a la presencia de BLEE, así como a la presencia de otras beta lactamasas plasmídicas o cromosomales. Al hacer el análisis de la resistencia a ceftriaxona con sensibilidad a cefotetan, se deduce que las beta lactamasas responsables de la resistencia a cefalosporinas de tercera generación deben ser primordialmente BLEE en $E$. coli y $K$. pneumoniae, mientras que deben ser secundarias a la presencia de AmpC en E. cloacae (8). Durante el año 2002 se identificó en Colombia la presencia de BLEE de tipo CTX-M en algunos de los hospitales de la red (23), lo que implica que este tipo de enzimas pueden tener una alta circulación entre nuestros hospitales, especialmente en la UCI. Uno de los factores más importantes para la aparición y mantenimiento de este tipo de enzimas es el uso de cefalosporinas de tercera generación (24). El aislamiento de los pacientes expuestos a cefalosporinas de tercera generación (ceftriaxona, ceftazidima, cefotaxima), así como aquellos colonizados o infectados por microorganismos con este patrón de resistencia debe recomendarse para evitar su diseminación en el hospital (24).

También se encontró una alta resistencia a las quinolonas, lo que limita su uso como agentes terapéuticos efectivos en la UCI, tanto en infecciones severas como en aquellas adquiridas sen el hospital. Al hacer el análisis de corresistencia con quinolonas y cefalosporinas de tercera generación (datos no mostrados), se encontró una alta resistencia a la ciprofloxacina y una baja 
tasa de corresistencia en E. coli, una tasa intermedia de corresistencia (7,9 \%) y una mayor tasa de resistencia a cefalosporinas de tercera generación en K. pneumoniae y una alta corresistencia (37 \%) con una alta resistencia a cefalosporinas de tercera generación en E. cloacae; estos datos sugieren que la resistencia a la ciprofloxacina puede ser un fenómeno independiente en $E$. coli, lo que puede explicarse por el amplio uso de quinolonas en la consulta externa y, quizás en muchos casos, incluso sin evaluación médica. Estudios realizados en aislamientos de Klebsiella de varios países sugieren que estas tasas de resistencia pueden estar relacionadas y que el uso previo de cefalosporinas de amplio espectro es un factor de riesgo para la aparición de resistencia a ciprofloxacina (25).

La susceptibilidad de los Gram negativos a los carbapenémicos es alta pero no está por encima de $99 \%$. Teniendo en cuenta que en nuestro país no se ha informado de la presencia de aislamientos de E. coli o Klebsiella spp. productores de carbapenemasas, estos datos deben ser interpretados con cautela, requiriendo de un mayor control de calidad para asegurar esta información. Además se debe llevar al extremo la vigilancia de aislamientos con este perfil de resistencia, con el fin de confirmar este comportamiento y, eventualmente, estudiarlo a través de técnicas moleculares.

Las tasas de resistencia de $P$. aeruginosa a la mayoría de antibióticos disponibles se ha incrementado durante los últimos años. Los datos del programa de vigilancia de RA en Unidades de Cuidado Intensivo de Europa y EEUU demuestran que la resistencia a los carbapenémicos, cefepime y la piperacilina tazobactam se acerca a $20 \%$, mientras que otros antibióticos tienen tasas de resistencias aún más altas $(19,26-29)$. El número de aislamientos probados contra meropenem durante el año 2002 fue pequeño, por lo que es difícil determinar si hubo un salto tan importante en la resistencia, o simplemente el número limitado de aislamientos no es capaz de reflejar el verdadero nivel de resistencia. El estudio MYSTIC mostró que la prevalencia de este tipo de microorganismos varía de forma importante en los países europeos, oscilando entre menos de $3 \%$ y 50 \% (30). Estos clones deben ser detectados oportunamente por los laboratorios clínicos y por los comités de infecciones intrahospitalarias de forma que se pueda evitar su diseminación, especialmente cuando estos pacientes tienen alternativas terapéuticas limitadas en nuestro país (31), y más aún cuando recientemente se describe en nuestro país la aparición de carbapenemasas (32). La frecuencia y resistencia de A. baumanni creció durante los años estudiados en todos los hospitales y especialmente se ha incrementado el número de gérmenes multiresistentes (27). Este fenómeno se ha observado en UCI de todo el mundo, especial- 
mente asociado al uso de cefalosporinas de tercera generación (33). La resistencia a imipenem es además seleccionada por el uso de este antibiótico.

La resistencia bacteriana es favorecida en las UCI por el uso amplio de antibióticos $(6,17,34,35)$. Cambios significativos en el uso de antibióticos pueden afectar la susceptibilidad antimicrobiana de los microorganismos encontrados en la UCI $(6,17,36,37)$. Por tanto estos resultados deben alertar acerca del uso de los antibióticos en nuestras UCI, el cual debe ser racional y prudente. Por otra parte, se debe estimular la vigilancia activa del consumo de antibióticos como una medida adicional para la vigilancia y control de la RA. Algunas estrategias de restricción de la prescripción de antibióticos, especialmente en UCI, han demostrado ser capaces además de disminuir el grado de RA, disminuir costos de la atención, tanto en países desarrollados con en vías de desarrollo $(38,39)$. Estas estrategias, sumadas a un mejoramiento en las medidas de control de infección deben ser recomendadas en nuestros hospitales como una de las medidas para el control de la RA.

Las limitaciones de un estudio de vigilancia de este tipo se deben considerar en el momento de interpretar los datos. Aunque se realizó un control de calidad de los laboratorios participantes los datos muestran que algunos perfiles antimicrobianos "inusuales" pueden estar pasando desapercibidos. Sin embargo, los datos mostrados son muy consistentes en cuanto a las tendencias observadas. Por otra parte, los resultados de este estudio son de gérmenes causantes de colonización e infección, por lo que deben ser analizados con precaución en el momento de decidir la prescripción de un paciente determinado; no obstante, para fines de vigilancia y establecimiento de medidas de control son muy útiles ya que reflejan mejor la ecología de las UCI porque a su vez puede relacionar el riesgo de transmisión cruzada (12). Para contener la diseminación de la RA, se debe tener en cuenta todos los gérmenes circulantes independientemente si están asociados a una colonización o a una infección.

Los resultados de este estudio son una invitación para el establecimiento rápido de políticas locales y regionales que contribuyan a la contención de la resistencia.

Agradecimientos: Grupo para el Control de la Resistencia Bacteriana (GREBO). Hospitales e Instituciones participantes (2001-2003): Universidad Nacional de Colombia (Aura Lucía Leal Castro, Javier Eslava Schmalbach, Giancarlo Buitrago Gutiérrez, Carlos Saavedra Trujillo), Clínica del Niño (Mauricio Luna, Tailandia Rodríguez, Martha Uzeta), Clínica de Occidente (Elkin Lemos, Martha Salinas), Clínica San Pedro Claver (Carlos Alquichire, Martha Ruiz), Fundación Cardioinfantil 
(Álvaro Arango, Patricia Bravo), Fundación Universitaria San Carlos (Jorge A. Cortés, Jaime Saravia), Hospital El Tunal (Elkin Lemos, Narda Olarte, Martha Garzón), Hospital Militar Central (Matilde Méndez, Carlos Pérez), Hospital de Occidente Kennedy (Elkin Lemos, Romelia Villa), Hospital San José (Claudia Fajardo, Paola Jiménez), Hospital Santa Clara (Gloria Inés Gallo, Luzmila López, Roberto Támara), Hospital Simón Bolívar (Carlos Álvarez, Gustavo Aristizábal, Constanza Correa), Hospital Universitario San Ignacio (Carlos Álvarez, Diana Moncada), Hospital Universitario Clínica San Rafael (Clemencia Ávila, Carlos Saavedra, Martha Pulido), Instituto Nacional de Cancerología (Patricia Arroyo, Jorge A. Cortés y Sonia I. Cuervo), Asociación Colombiana de Infectología (ACIN) - Capítulo Central e Instituto Nacional de Salud.

\section{REFERENCIAS}

1. Jones RN. Resistance patterns among nosocomial pathogens: trends over the past few years. Chest 2001;119(2 Suppl):397S-404S.

2. Diekema DJ, Pfaller MA, Schmitz FJ, Smayevsky J, Bell J, Jones RN, et al. Survey of infections due to Staphylococcus species: frequency of occurrence and antimicrobial susceptibility of isolates collected in the United States, Canada, Latin America, Europe, and the Western Pacific region for the SENTRY Antimicrobial Surveillance Program, 1997-1999. Clin Infect Dis 2001;32 Suppl 2:S114-32.

3. Sader HS, Jones RN, Andrade-Baiocchi S, Biedenbach DJ. Four-year evaluation of frequency of occurrence and antimicrobial susceptibility patterns of bacteria from bloodstream infections in Latin American medical centers. Diagn Microbiol Infect Dis 2002;44(3):273-80.

4. Kollef MH, Fraser VJ. Antibiotic resistance in the intensive care unit. Ann Intern Med 2001;134(4):298-314.

5. Neuhauser MM, Weinstein RA, Rydman R, Danziger LH, Karam G, Quinn JP. Antibiotic resistance among gram-negative bacilli in US intensive care units: implications for fluoroquinolone use. Jama 2003;289(7):885-8.

6. Alvarez CA. Control de la resistencia bacteriana. In: Gomez A, Alvarez CA, Leon A, editors. Enfermedades infecciosas en UCI. Una aproximacion basada en la evidencia. Bogota: Distribuna, 2003:447 - 480.

7. Lucet JC, Chevret S, Durand-Zaleski I, Chastang C, Regnier B. Prevalence and risk factors for carriage of methicillin-resistant Staphylococcus aureus at admission to the intensive care unit: results of a multicenter study. Arch Intern Med 2003;163(2):181-8.

8. Livermore DM, Winstanley TG, Shannon KP. Interpretative reading: recognizing the unusual and inferring resistance mechanisms from resistance phenotypes. J Antimicrob Chemother 2001;48 Suppl 1:87-102.

9. Leal AL, Buitrago G, Alvarez CA, Eslava J. Creación de una red de vigilancia de la resistencia bacteriana en Bogotá. En prensa 2005.

10. Simonsen GS, Tapsall JW, Allegranzi B, Talbot EA, Lazzari S. The antimicrobial resistance containment and surveillance approach--a public health tool. Bull World Health Organ 2004;82(12):928-34. 
11. Singh N, Yu VL. Rational empiric antibiotic prescription in the ICU. Chest 2000;117(5):1496-9.

12. Fridkin SK, Steward CD, Edwards JR, Pryor ER, McGowan JE, Jr., Archibald LK, et al. Surveillance of antimicrobial use and antimicrobial resistance in United States hospitals: project ICARE phase 2. Project Intensive Care Antimicrobial Resistance Epidemiology (ICARE) hospitals. Clin Infect Dis 1999;29(2):245-52.

13. Fluit AC, Verhoef J, Schmitz FJ. Frequency of isolation and antimicrobial resistance of gram-negative and gram-positive bacteria from patients in intensive care units of 25 European university hospitals participating in the European arm of the SENTRY Antimicrobial Surveillance Program 19971998. Eur J Clin Microbiol Infect Dis 2001;20(9):617-25.

14. Ponce de Leon-Rosales SP, Molinar-Ramos F, Dominguez-Cherit G, RangelFrausto MS, Vazquez-Ramos VG. Prevalence of infections in intensive care units in Mexico: a multicenter study. Crit Care Med 2000;28(5):131621.

15. Vincent JL, Bihari DJ, Suter PM, Bruining HA, White J, Nicolas-Chanoin MH, et al. The prevalence of nosocomial infection in intensive care units in Europe. Results of the European Prevalence of Infection in Intensive Care (EPIC) Study. EPIC International Advisory Committee. Jama 1995;274(8):639-44.

16. Arias CA, Reyes J, Zuniga M, Cortes L, Cruz C, Rico CL, et al. Multicentre surveillance of antimicrobial resistance in enterococci and staphylococci from Colombian hospitals, 2001-2002. J Antimicrob Chemother 2003;51(1):5968.

17. National Nosocomial Infections Surveillance (NNIS) System Report, data summary from January 1992 through June 2003, issued August 2003. Am J Infect Control 2003;31(8):481-98.

18. Jarlier V, Fosse T, Philippon A. Antibiotic susceptibility in aerobic gram-negative bacilli isolated in intensive care units in 39 French teaching hospitals (ICU study). Intensive Care Med 1996;22(10):1057-65.

19. Streit JM, Jones RN, Sader HS, Fritsche TR. Assessment of pathogen occurrences and resistance profiles among infected patients in the intensive care unit: report from the SENTRY Antimicrobial Surveillance Program (North America, 2001). Int J Antimicrob Agents 2004;24(2):111-8.

20. Mendes C, Hsiung A, Kiffer C, Oplustil C, Sinto S, Mimica I, et al. Evaluation of the in vitro activity of 9 antimicrobials against bacterial strains isolated from patients in intensive care units in brazil: MYSTIC Antimicrobial Surveillance Program. Braz J Infect Dis 2000;4(5):236-44.

21. Panesso D, Ospina S, Robledo J, Vela MC, Pena J, Hernandez O, et al. First characterization of a cluster of VanA-type glycopeptide-resistant Enterococcus faecium, Colombia. Emerg Infect Dis 2002;8(9):961-5.

22. Jones RN, Salazar JC, Pfaller MA, Doern GV. Multicenter evaluation of antimicrobial resistance to six broad-spectrum beta-lactams in Colombia using the Etest method. The Colombian Antimicrobial Resistance Study Group. Diagn Microbiol Infect Dis 1997;29(4):265-72. 
23. Villegas MV, Correa A, Perez F, Zuluaga T, Radice M, Gutkind G, et al. CTXM-12 beta-lactamase in a Klebsiella pneumoniae clinical isolate in Colombia. Antimicrob Agents Chemother 2004;48(2):629-31.

24. Paterson DL, Ko WC, Von Gottberg A, Mohapatra S, Casellas JM, Goossens H, et al. International prospective study of Klebsiella pneumoniae bacteremia: implications of extended-spectrum beta-lactamase production in nosocomial Infections. Ann Intern Med 2004;140(1):26-32.

25. Paterson DL, Mulazimoglu L, Casellas JM, Ko WC, Goossens H, Von Gottberg A, et al. Epidemiology of ciprofloxacin resistance and its relationship to extended-spectrum beta-lactamase production in Klebsiella pneumoniae isolates causing bacteremia. Clin Infect Dis 2000;30(3):473-8.

26. Unal S, Masterton R, Goossens H. Bacteraemia in Europe--antimicrobial susceptibility data from the MYSTIC surveillance programme. Int J Antimicrob Agents 2004;23(2):155-63.

27. Friedland I, Stinson L, Ikaiddi M, Harm S, Woods GL. Phenotypic antimicrobial resistance patterns in Pseudomonas aeruginosa and Acinetobacter: results of a Multicenter Intensive Care Unit Surveillance Study, 1995-2000. Diagn Microbiol Infect Dis 2003;45(4):245-50.

28. Glupczynski Y, Delmee M, Goossens H, Struelens M. A multicentre survey of antimicrobial resistance in gram-negative isolates from Belgian intensive care units in 1994-1995. Belgian Multicenter ICU Study Group. Acta Clin Belg 1998;53(1):28-38.

29. Garcia-Rodriguez JA, Jones RN. Antimicrobial resistance in gram-negative isolates from European intensive care units: data from the Meropenem Yearly Susceptibility Test Information Collection (MYSTIC) programme. J Chemother 2002;14(1):25-32.

30. Goossens H. Susceptibility of multi-drug-resistant Pseudomonas aeruginosa in intensive care units: results from the European MYSTIC study group. Clin Microbiol Infect 2003;9(9):980-3.

31. Markou N, Apostolakos H, Koumoudiou C, Athanasiou M, Koutsoukou A, Alamanos I, et al. Intravenous colistin in the treatment of sepsis from multiresistant Gram-negative bacilli in critically ill patients. Crit Care 2003;7(5):R78-83.

32. Crespo MP, Woodford N, Sinclair A, Kaufmann ME, Turton J, Glover J, et al. Outbreak of carbapenem-resistant Pseudomonas aeruginosa producing VIM-8, a novel metallo-beta-lactamase, in a tertiary care center in Cali, Colombia. J Clin Microbiol 2004;42(11):5094-101.

33. Lee SO, Kim NJ, Choi SH, Hyong Kim T, Chung JW, Woo JH, et al. Risk factors for acquisition of imipenem-resistant Acinetobacter baumannii: a casecontrol study. Antimicrob Agents Chemother 2004;48(1):224-8.

34. Monnet DL, Archibald LK, Phillips L, Tenover FC, McGowan JE, Jr., Gaynes RP. Antimicrobial use and resistance in eight US hospitals: complexities of analysis and modeling. Intensive Care Antimicrobial Resistance Epidemiology Project and National Nosocomial Infections Surveillance System Hospitals. Infect Control Hosp Epidemiol 1998;19(6):388-94. 
35. Meyer E, Schwab F, Jonas D, Rueden H, Gastmeier P, Daschner FD. Surveillance of antimicrobial use and antimicrobial resistance in intensive care units (SARI): 1. Antimicrobial use in German intensive care units. Intensive Care Med 2004;30(6):1089-96.

36. Gentry C, Flournoy DJ, Reinert R. Analysis of antimicrobial resistance among gram-negative bacilli and antimicrobial use in intensive care unit patients for 5 years in a Veterans Affairs medical center. Am J Infect Control 2002;30(7):411-6.

37. Itokazu GS, Quinn JP, Bell-Dixon C, Kahan FM, Weinstein RA. Antimicrobial resistance rates among aerobic gram-negative bacilli recovered from patients in intensive care units: evaluation of a national postmarketing surveillance program. Clin Infect Dis 1996;23(4):779-84.

38. Dos Santos EF, Silva AE, Pinhati HM, Maia Mde O. Effectiveness of the actions of antimicrobial control in the intensive care unit. Braz $\mathrm{J}$ Infect Dis 2003;7(5):290-6.

39. Geissler A, Gerbeaux P, Granier I, Blanc P, Facon K, Durand-Gasselin J. Ra tional use of antibiotics in the intensive care unit: impact on microbial resistance and costs. Intensive Care Med 2003;29(1):49-54. 